\title{
RESEARCH
}

Open Access

\section{The role of interferon regulatory factor 8 for retinal tissue homeostasis and development of choroidal neovascularisation}

Peipei Zhang ${ }^{1}$, Anja Schlecht ${ }^{1,2}$, Julian Wolf ${ }^{1}$, Stefaniya Boneva ${ }^{1}$, Yannik Laich¹, Jana Koch ${ }^{1}$, Franziska Ludwig ${ }^{1}$, Myriam Boeck', Adrian Thien ${ }^{1}$, Carmen Härdtner ${ }^{3,4}$, Katrin Kierdorf ${ }^{5,6,7}$, Hansjürgen Agostini ${ }^{1}$, Günther Schlunck', Marco Prinz $z^{5,7,8}$, Ingo Hilgendorf ${ }^{3,4}$, Peter Wieghofer ${ }^{5,9^{*}+}$ and Clemens Lange ${ }^{1 *+}$ (D)

\begin{abstract}
Background: Microglia cells represent the resident innate immune cells of the retina and are important for retinal development and tissue homeostasis. However, dysfunctional microglia can have a negative impact on the structural and functional integrity of the retina under native and pathological conditions.

Methods: In this study, we examined interferon-regulatory factor 8 (Irf8)-deficient mice to determine the transcriptional profile, morphology, and temporospatial distribution of microglia lacking Irf8 and to explore the effects on retinal development, tissue homeostasis, and formation of choroidal neovascularisation (CNV).

Results: Our study shows that Irf8-deficient MG exhibit a considerable loss of microglial signature genes accompanied by a severely altered MG morphology. An in-depth characterisation by fundus photography, fluorescein angiography, optical coherence tomography and electroretinography revealed no major retinal abnormalities during steady state. However, in the laser-induced CNV model, Irf8-deficient microglia showed an increased activity of biological processes critical for inflammation and cell adhesion and a reduced MG cell density near the lesions, which was associated with significantly increased CNV lesion size.

Conclusions: Our results suggest that loss of Irf8 in microglia has negligible effects on retinal homeostasis in the steady state. However, under pathological conditions, Irf8 is crucial for the transformation of resident microglia into a reactive phenotype and thus for the suppression of retinal inflammation and CNV formation.
\end{abstract}

Keywords: Irf8, Interferon regulatory factor 8, Retinal microglia, Choroidal neovascularisation, RNA sequencing

\footnotetext{
* Correspondence: peter.wieghofer@medizin.uni-leipzig.de;

clemens.lange@uniklinik-freiburg.de

${ }^{+}$Peter Wieghofer and Clemens Lange both contributed equally to the work.

${ }^{5}$ Medical Faculty, Institute of Neuropathology, University Hospital, University

of Freiburg, Freiburg im Breisgau, Germany

${ }^{1}$ Medical Faculty, Eye Center, University Hospital, University of Freiburg,

Freiburg im Breisgau, Germany

Full list of author information is available at the end of the article
}

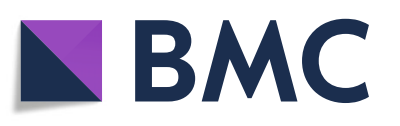

(- The Author(s). 2021 Open Access This article is licensed under a Creative Commons Attribution 4.0 International License, which permits use, sharing, adaptation, distribution and reproduction in any medium or format, as long as you give appropriate credit to the original author(s) and the source, provide a link to the Creative Commons licence, and indicate if changes were made. The images or other third party material in this article are included in the article's Creative Commons licence, unless indicated otherwise in a credit line to the material. If material is not included in the article's Creative Commons licence and your intended use is not permitted by statutory regulation or exceeds the permitted use, you will need to obtain permission directly from the copyright holder. To view a copy of this licence, visit http://creativecommons.org/licenses/by/4.0/. The Creative Commons Public Domain Dedication waiver (http://creativecommons.org/publicdomain/zero/1.0/) applies to the data made available in this article, unless otherwise stated in a credit line to the data. 


\section{Background}

Retinal microglia cells (rMG) constitute the resident myeloid cell population in the neuroretina and are critical for retinal development, tissue homeostasis and response to cell damage. During development, rMG are in intimate contact to endothelial tip cells and contribute to postnatal vascular development [16] as well as neuronal survival by modulating programmed cell death and trophic influences [56]. During adulthood, rMG interact closely with synapses to maintain synaptic structure and electroretinal function and continuously scan the local environment for danger signals associated with injury or pathogens [56]. In response to tissue damage or infection, rMG rapidly attain an activated phenotype, migrate towards the site of injury and contribute to phagocytosis, inflammation and pathological events [4, 52, 59]. As such, activated microglia cells have been found in the subretinal space of patients with age-related macular degeneration (AMD) and in particular at sites of choroidal neovascularisation $(\mathrm{CNV})$ in neovascular $\operatorname{AMD}[11,20]$ which is a common cause of irreversible blindness in the elderly $[10,60]$. Studies on the role of rMG in the development of $\mathrm{CNV}$, however, revealed conflicting results, and both detrimental and protective roles of MG for the progression of CNV have been discussed in the past [2, $14,36,49,51]$.

The interferon regulatory factor (IRF) family of transcription factors consists of nine members that are involved in hematopoietic differentiation, oncogenesis, Toll-like and purinergic receptor signalling and expression of interferons and interferon-inducible genes [39, 57]. In particular, Irf8 plays a pivotal role in the regulation of lineage commitment and MG cell maturation during brain development [27, 47]. Besides its essential role during development, Irf8 is crucial for the function of resident myeloid cells in the adult steady state. As such, the deletion of Irf 8 in mice leads to a disturbed homeostasis of resident tissue macrophages in the liver, the kidney and brain including microglia and other CNS-associated macrophages $[19,25,48,58]$. The role of Irf8 in regulating rMG gene expression and its influence on retinal development and neuroretinal function, however, are currently unknown.

The aim of this study was to determine the function of IRF8 in retinal microglia in the healthy as well as perturbed retina. Specifically, we aimed to investigate whether IRF8 is involved in microglial cell homeostasis, neuroretinal function and pathological CNV formation. The latter is of particular interest, as PU.1 and CSF1R signalling acting upstream and downstream of IRF8, respectively, are critical for postnatal angiogenesis and formation of pathological neovascularisation in the eye [16, 54]. For this purpose, we analysed Irf8 reporter and knockout mice by in vivo imaging, functional studies, flow cytometry, immunohistochemistry and RNA sequencing (RNA-seq). We found that Irf8-deficient MG exhibited functionally relevant alterations in gene expression patterns that were associated with a significant disruption of microglial development, normal postnatal retinal vascular and functional development, and increased CNV lesion size in the adult situation.

\section{Methods}

Mice

All animal experiments were authorized by the local animal care and use committee under the respective EU, national, federal and institutional regulations for animal experiments (ethical protocol numbers G14/89, G20/13). Mice were bred on a C57BL/6J background and devoid of the Crb1 mutation. $\mathrm{C} x 3 \mathrm{cr} 1^{\mathrm{GFP} / \mathrm{GFP}}$ mice were crossed with C57BL/6J mice to generate $\mathrm{Cx} 3 \mathrm{cr} 1^{\mathrm{GFP} /+}$ (Irf8 WT) mice. $\mathrm{Irfs}^{-/-}$mice were crossed with $\mathrm{Irfs} 8^{-1-} \mathrm{Cx} 3 \mathrm{crl}^{\mathrm{GFP} / \mathrm{GFP}}$ mice to obtain $\mathrm{Cx} 3 \mathrm{cr} 1^{\mathrm{GFP} /+}: \operatorname{Irf}^{-/-}$(Irf8 KO) mice [24, 26]. Phage artificial chromosome-transgenic Irf8-VENUS reporter mice were used to trace the expression of IRF8 [53]. CAG::mRFP1 mice were purchased from the Jackson Laboratory (Bar Harbor, ME).

\section{Genotyping}

Transgenic mice were genotyped according to the primers and programs shown in supplemental table 1.

\section{Laser-induced choroidal neovascularisation (CNV)}

The laser-induced CNV model was used as previously described $[15,31,50]$. In brief, mice were anaesthetized by intraperitoneal administration of ketamine hydrochloride $(100 \mathrm{mg} / \mathrm{kg}$, Pharmacia \& Upjohn, Erlangen, Germany) and xylazine (6 mg/kg, Bayer Vital GmbH, Leverkusen, Germany). Pupillary dilatation was achieved by applying 0.5\% tropicamide (Bausch + Lomb, Berlin, Germany) and 5\% phenylephrine hydrochloride (URSAPHARM Arzneimittel GmbH, Saarbrücken, Germany). After covering the cornea with a coverslip coated with dexpanthenol eye gel $(50 \mathrm{mg} / \mathrm{g}$, Bausch + Lomb, Berlin, Germany), three to six laser spots $(488 \mathrm{~nm}, 150 \mathrm{~mW}$, $100 \mu \mathrm{m}$ and $100 \mathrm{~ms}$ ) were applied to each eye using the VISULAS 532s Laser System (Carl Zeiss, Jena, Germany) in combination with ZEISS Laser Slit Lamp 532s (Carl Zeiss, Jena, Germany). Only laser spots with visible formation of vaporisation bubbles were included in this study.

\section{Bone marrow transplantation}

Bone marrow transplantation experiments were carried out as previously described [22]. In brief, a total of 12 recipient control and 14 Irf8 knockout mice were headshielded and lethally irradiated (RS2000 irradiator, Rad Source, Kanas, USA) in two independent experiments. 
Meanwhile, bone marrow cells (BMCs) were collected from the tibias and femurs of CAG-mRFP1 mice and resuspended in phosphate-buffered saline (PBS). The recipient mice were intravenously injected with $3 \times 10^{6}$ $\mathrm{BMCs}$ via the tail vein. Nine weeks after bone marrow transplantation, the efficiency of reconstitution was assessed by flow cytometry which will be explained below.

\section{In vivo characterisation and analysis}

Fundus morphology, retinal structure and physiological function were investigated using fundus photography, fundus fluorescein angiography (FFA), optical coherence tomography (OCT) and electroretinography (ERG) as previously described [32]. Fundus photography, FFA and OCT were performed using a Micron III retinal microscope (Phoenix Technology Group, Pleasanton, CA, USA) and the StreamPix software (Norpix Inc., Montreal, Canada). For FFA, 10\% sodium fluorescein (Alcon, Freiburg, Germany) was diluted to a concentration of 50 $\mu \mathrm{L} / \mathrm{mL}$ in $0.9 \%$ sodium chloride for injection (VWR, Leuven, Belgium) and administered intraperitoneally (2 $\mu \mathrm{L} / \mathrm{g})$. Ninety seconds after dye injection, the angiograms were recorded. For quantification of CNV size, hyperfluorescent areas in early-phase angiograms were measured in pixels using ImageJ. Image-guided OCT was performed using the OCT2 scan head. In OCT images, the thickness of the inner nuclear layer (INL) (200 pixels from the optic nerve head) was measured using ImageJ (https://imagej.nih.gov/ij/). For ERG, mice were darkadapted overnight and anaesthetized by intraperitoneal injection of ketamine hydrochloride $(66.8 \mathrm{mg} / \mathrm{kg})$ and xylazine $(12.76 \mathrm{mg} / \mathrm{kg})$. ERG signals were amplified, recorded and analysed automatically using Ganzfeld Q450 (Roland-Consult, Brandenburg, Germany) with the integrated software developed by Prof. Dr. rer. nat. Michael Bach (Eye Center, University of Freiburg, Germany).

\section{Immunohistochemistry and imaging}

After intracardiac perfusion with PBS and 4\% paraformaldehyde (PFA), eyes were fixated in 4\% PFA for 45 $\mathrm{min}$ at room temperature and processed to RPEchoroidal-scleral and retinal flat mounts. After incubation in PBST/BSA blocking buffer overnight, the flat mounts were incubated with primary antibodies against collagen type IV (1:500, AB769, Merck Millipore, Darmstadt, Germany), Iba1 (1:500, \#019-19741, Wako, Neuss, Germany) or alpha smooth muscle actin (SMA, 1:500, ab5694, Abcam, Cambridge, UK) for two nights at $4{ }^{\circ} \mathrm{C}$, followed by incubation with Alexa Fluor 568 or 647 conjugated secondary antibodies overnight at $4{ }^{\circ} \mathrm{C}$ (1: 500, Life technologies, Eugene, OR, USA). Eyes of Irf8VENUS mice were fixated in 4\% PFA for $1 \mathrm{~h}$ and incubated in $10 \%, 20 \%$ and $30 \%$ sucrose for $24 \mathrm{~h}$ each prior to embedding in Tissue-Tek O.C.T. compound (Sakura, Aplphen aan den Rijn, The Netherlands). Seven micrometre-thick cryosections were cut using a cryostat (Leica CM1950, Leica, Nussloch, Germany). Following blocking in Ultra $\mathrm{V}$ block for $10 \mathrm{~min}$ at room temperature, the sections were incubated with primary antibodies against ßIII tubulin (1:500, ab18207, Abcam, Cambridge, UK), collagen type IV (1:1000, ab6586, Abcam, Cambridge, UK), Ceh-10 homeo domain containing homolog (CHX10, 1:200, ab16141, Abcam, Cambridge, UK), GFP (1:500, 600-101-215, ROCKLAND, Limerick, PA, USA), glial fibrillary acidic protein (GFAP, 1:500, 087A1005RE, Fremont, CA, USA) or Iba1 (1:500) for 60 min with corresponding Alexa Fluor ${ }^{\circ}$ 568conjugated secondary antibodies (1:500, Life technologies, Eugene, OR, USA). Nuclei were counterstained with 4,6-diamidino-2-phenylindole (DAPI). Stainings were imaged using the Nano Zoomer S60 digital slide scanner (Hamamatsu, Herrsching am Ammersee, Germany) and analysed with NDP viewer software (Hamamatsu, Herrsching am Ammersee, Germany) or with a confocal laser scanning microscope (Zeiss LSM 510 or Leica TCS SP8 or Olympus FV1000), Zen software (Carl Zeiss, Jena, Germany), LAS X software (Leica, Nussloch, Germany) or Fluoview FV1000 (Olympus, Tokyo, Japan). For a detailed list of antibodies used, see supplementary table 2.

\section{Three-dimensional reconstruction of retinal microglia}

Imaging for 3D reconstruction was performed using a Zeiss LSM 510 confocal laser scanning microscope with a $20 \times$ objective, $3 \times$ zoom and $1024 \times 1024$ pixel resolution. The interval thickness of the z-stacks was set to $1.0 \mu \mathrm{m}$. The morphology of retinal microglia in the inner plexiform layer (IPL) and outer plexiform layer (OPL) was determined by a three-dimensional reconstruction using the filament mode of IMARIS software (Bitplane, Zurich, Switzerland). Three cells per layer and mouse were reconstructed and analysed.

\section{Fluorescence-activated cell sorting}

Following transcardial perfusion with $1 \times \mathrm{PBS}$ and enucleation, eyes were dissected in ice-cold $1 \times$ PBS to isolate the retinae of $\operatorname{Irf}^{+/+} \mathrm{C} \times 3 \mathrm{crr} 1^{\mathrm{GFP} /+}$ or Irf $8^{-/-} \mathrm{C} x 3 \mathrm{cr} 1^{\mathrm{GFP} /+}$. For the lasered mice, the central parts $(70 \%)$ of the retinae were used for FACS while the peripheral parts were omitted. After tissue homogenisation and filtration through a $50-\mu \mathrm{m}$ cell strainer (Sysmex, Goerlitz, Germany), dead cell exclusion was performed by incubation with fixable viability dye 780 (1:1000, 650865-14, eBioscience, Waltham, MA, USA). Anti-CD16/ CD32 (Fc) receptor (1:200, 553142, BD Biosciences, Heidelberg, Germany) was used to avoid unspecific binding. Following staining with anti-CD45 (1:200, 103133, 
BioLegend, San Diego, CA, USA), anti-CD11b (1:200, 17-0112-83, eBioscience, Waltham, MA, USA), antiLy6C (1:200, 560593, BD Bioscience, Heidelberg, Germany) and anti-Ly6G (1:200, 560601, BD Biosciences, Heidelberg, Germany) for $20 \mathrm{~min}$ at $4{ }^{\circ} \mathrm{C}$, retinal microglia characterised as $\mathrm{CD} 45^{\text {low }} \mathrm{CD} 11 \mathrm{~b}^{+} \mathrm{C} \times 3 \mathrm{cr} 1^{\mathrm{GF}-}$ ${ }^{\mathrm{P} /+}$ Ly6C $\mathrm{Cy}^{-}$6G- were analysed and sorted into RNA stabilisation reagent (QIAGEN, Hilden, Germany) using a MoFlo Astrios EQ High Speed Cell Sorter (Beckman Coulter, Munich, Germany). For RNA sequencing, an average number of 10,000 retinal microglia per sample was obtained from pooling 3 to 4 mice. Flow cytometric purification of wild-type retinal and brain microglia together with bone marrow-derived monocytes was described before [59]. Data are available under the GSE accession number GSE160845. For a detailed list of antibodies used, see supplementary table 2 .

\section{RNA extraction}

RNA extraction, RNA library preparation and RNA sequencing were performed in collaboration with the Genomics Core Facility "KFB-Center of Excellence for Fluorescent Bioanalytics" (University of Regensburg, Germany). RNA extraction was performed according to manufacturer's instructions using the RNeasy Plus Mini Kit (QIAGEN, Hilden, Germany). After pelleting the sample by centrifugation, the RNA stabilisation reagent was removed and replaced by RLT Plus buffer for lysing retinal microglia. Genomic DNA was removed selectively and efficiently by using gDNA Eliminator spin columns for RNA purification. After adding Ethanol to the flow-through, the sample was applied to an RNeasy MinElute spin column to collect RNA. Finally, after washing the column, total purified RNA was eluted in RNase-free water. The quality and integrity of total RNA was assessed with a Agilent 2100 Bioanalyser in combination with the RNA 6000 Pico LabChip Kit (Agilent, Palo Alto, CA, USA).

\section{RNA sequencing}

First-strand cDNA was generated using SMARTer Ultra Low Input RNA Kit for Sequencing v4 (Clontech Laboratories, Inc., Mountain View, CA, USA). Doublestanded cDNA was amplified with LD PCR and purified with AMPure XP beads. Library preparation was constructed conforming to the Illumina Nextera XT Sample Preparation Guide (Illumina, San Diego, CA, USA). In brief, $150 \mathrm{pg}$ of input cDNA was tagmented via Nextera $\mathrm{XT}$ transposome. The products were purified and amplified with a limited-cycle PCR program to construct sequencing libraries. The libraries were quantified with the KAPA SYBR FAST ABI Prism Library Quantification Kit (Kapa Biosystems, Wobum, MA, USA). Equimolar amounts of each library were pooled for cluster generation on the cBot using the Illumina TruSeq SR Cluster Kit v3. The sequencing run was performed on a HiSeq1000 instrument with TruSeq SBS Kit v3 according to the Illumina HiSeq 1000 System User Guide. Illumina image analysis and base calling were recorded in library base call format (.bcl) and further converted to Fastq files via the CASAVA1.8.2 software.

\section{RNA sequencing data analysis}

Quality control and transcriptome profiling including reads mapping, annotation, quantification and normalisation were performed by GenXPro (GenXPro, Frankfurt, Germany). Briefly, FastQC was performed to assess sequencing quality. After removing reads containing adapter sequences and duplicate reads via cutadapt software (GitHub, San Francisco, CA, USA) and FastUniq, the filtered reads were mapped to the mouse genome from ENSEMBL (https://www.ensembl.org/Mus musculus/Info/Index) using bowtie2. The transcripts were functionally annotated with gene transfer format file version 90, quantified using HTSeq and normalised as transcripts per kilobase million (TPM) via DESeq2. Differential gene expression analysis with threshold (log2 fold change greater than 1.5 or less than $-1.5, p<$ $0.05, \mathrm{TPM} \geq 100$ in at least one of the two compared groups) was performed using DESeq2. Data was visualised using RStudio (v1.2.1335) and R (v3.5.3). Volcano plots were created using the ggplot 2 package, and Gene Ontology (GO) analysis was performed using $\mathrm{R}$ with the clusterProfiler 3.10.1 package [62].

\section{Protein analysis}

Protein was extracted from the choroid and the retinae using RIPA buffer (\#R2078, Sigma Aldrich) containing protease inhibitor (cOmplete, Mini; edta-FREE Protease inhibitor Cocktail, Roche Diagnostics, Manheim, Germany) and phosphatase inhibitors (PhosSTOP, Roche Diagnostics, Manheim, Germany), respectively, for preservation. Total protein concentration for each sample was measured with the Pier$\mathrm{ce}^{\mathrm{TM}}$ Bicinchoninic Acid Protein Assay Kit (Thermo Fisher Scienticis, Inc., Rockland, IL, USA).

\section{Statistical analysis}

Statistical analysis was performed using GraphPad Prism v6 (La Jolla, USA) as follows: an unpaired $t$ test was applied if the normality was given by the KolmogorovSmirnov test. Otherwise, the Mann-Whitney $U$ test was used. Difference with significance was defined as $p<0.05$.

\section{Results}

IRF8 is the most abundantly expressed member of the IRF family in retinal microglia

The interferon regulatory factor (IRF) family is critical for the development, maturation and function of 
myeloid cells [40]. Using flow cytometry and RNA sequencing, we first determined the expression levels of different IRF family members in adult retinal microglia (rMG), brain microglia (bMG) and bone marrow (BM) monocytes (Fig. 1A, B). In general, genes belonging to the IRF family were expressed at different levels in rMG, bMG and BM monocytes (Fig. 1B). Compared with other IRF family members, Irf5 and Irf8 exhibited the highest expression levels in rMG, bMG and BM monocytes. In the retina, Irf8 emerged as the most prominent IRF member in rMG. Interestingly, the expression of Irf8 in rMG exceeded the expression in bMG and BM monocytes indicating a distinct function of Irf8 for rMG. To validate Irf8 expression in adult rMG, we next analysed Irf8-VENUS reporter mice by flow cytometry and immunohistochemistry. Flow cytometry analysis revealed a strong VENUS expression $(98.7 \% \pm 0.5 \%)$ in
$\mathrm{CD} 45^{\mathrm{lo}} \mathrm{CD} 11 \mathrm{~b}^{+} \mathrm{rMG}$ in the steady state (Fig. $1 \mathrm{C}$ ). In line with this finding, immunofluorescence analysis of retinal flat mounts and cryosections confirmed that IRF8 is mostly expressed in IBA1-positive microglial cells in the steady state (Fig. 1D and Supplementary Figure 1C). Since expression of Irf family members may change in response to stress and inflammation, we next assessed the expression levels of all IRF family members (1-9) and of common MG signature genes, such as Tmem119 and P2ry12, in retinal MG in 2-3 months old $C \times 3 c r 1^{\mathrm{GFP} /+}$ mice in the steady state and upon MG activation in the laser-induced $\mathrm{CNV}$ model. While the expression of the signature genes P2ry 12 and Tmem 119 decreased in retinal MG after laser injury, activation markers such as $C d 74$ were increased, as reported before [59]. However, most members of the IRF family were expressed at the same level in the rMG in the context of

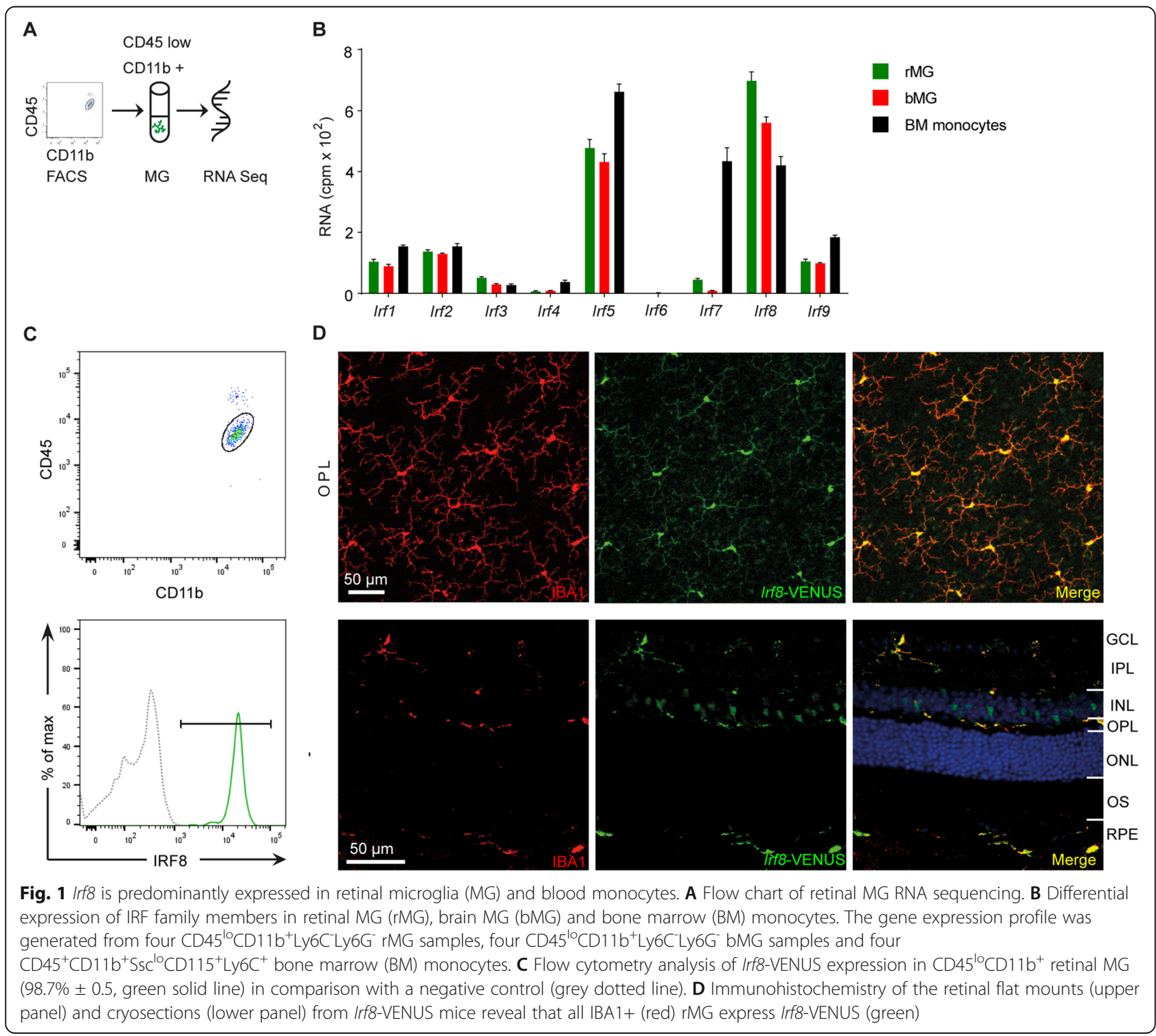


laser-induced inflammation compared with controls. Only Irf2 and Irf5 were slightly downregulated in retinal MG, whereas Irf7 was modestly upregulated. Notably, the expression of Irf8 in retinal MG remained stable in the laser-induced $\mathrm{CNV}$ model compared with controls (data not shown).

\section{Deficiency of Irf8 has a substantial impact on morphology, distribution and transcriptional activity of retinal MG in the steady state}

To assess the role of IRF8 in distribution and cell morphology of rMG in the adult situation, we next examined retinal flat mounts from 8-week-old Irf8 knockout (KO) mice by immunofluorescence microscopy (Fig. 2A,B). Irf8 $\mathrm{KO}$ mice revealed a strikingly altered $\mathrm{rMG}$ distribution and morphology compared with wild-type (WT) animals, characterised by slightly reduced MG numbers in the inner plexiform layer (IPL, Irf8 WT: $104 \pm 7$ cells $/ \mathrm{mm}^{2}$, Irf8 KO: $88 \pm 5$ cells $/ \mathrm{mm}^{2}, p=0.09$ ) and highly significant decrease of cell numbers in the outer plexiform layer (OPL, Irf8 WT: $116 \pm 6$ cells $/ \mathrm{mm}^{2}$; Irf8 KO: $43 \pm 1$ cells/ $\mathrm{mm}^{2}, p<0.0001$, Fig. 2B,C). Quantitative morphometric analysis using IMARIS revealed a severely altered morphology of retinal microglia in Irf8 $\mathrm{KO}$ mice, including significantly shorter length of dendrites (IPL: Irf8 KO: 356.5 $\pm 14.3 \mu \mathrm{m}$, Irf8 WT $1043.0 \pm 59.4 \mu \mathrm{m}, p=0.009$; OPL: Irf8 KO: $297.7 \pm 35.6 \mu \mathrm{m}$, Irf8 WT: $788.8 \pm 26.1 \mu \mathrm{m}, p=$ 0.01 ), and reduced number of dendrite segments (IPL: Irf8 KO: $62 \pm 4$, Irf8 WT: $167 \pm 14, p=0.009$; OPL: Irf8 KO: $44 \pm 6$, Irf8 WT: $139 \pm 6, p=0.01$ ), branch points (IPL: Irf8 KO: $30 \pm 2, \operatorname{Irf} 8$ WT: $82 \pm 7,, p=0.009$; OPL: $\operatorname{Irf} 8$ KO: $21 \pm 3$, Irf8 WT: $68 \pm 3, p=0.01$ ) and terminal points (IPL: Irf8 KO: $33 \pm 2$, Irf8 WT: $86 \pm 7, p=0.009$; OPL: Irf8 KO: $23 \pm 3$, Irff WT: $71 \pm 3, p=0.01$, Fig. $2 \mathrm{D}, \mathrm{E})$ compared with controls. Having established a profoundly altered rMG distribution and phenotype in the adult situation, we next explored rMG cell numbers at earlier stages of postnatal development. Interestingly, reduced microglia cell numbers were already present in the neuroblast layer at postnatal day 1 (P1) and later in the OPL at P7 but only transiently in the IPL at P7 that could be compensated until adulthood (Suppl. Figure 2A, B). These findings suggest an impaired MG distribution specifically in the deeper layers of the retina which is already present shortly after birth and persists into adulthood.

To explore the associated transcriptional changes in Irf8-deficient rMG, we next performed RNA sequencing (RNA-seq) of FACS-sorted rMG from adult Irf8 KO mice and controls. We determined 277 differentially expressed genes (DEGs) of which 142 were significantly upregulated, and 135 genes were downregulated in Irf8 KO microglia compared with microglia of control mice (Fig. 3A). Gene ontology (GO) cluster analysis revealed that most of these downregulated genes contribute to processes such as "cell migration" (GO:0016477, p.adj. < $1.3 \times 10^{-5}$ ), "cell motility" (GO:0048870, p.adj. < $4.8 \times$ $10^{-5}$ ), "localization of cells" (GO:0051674, p.adj. < $4.8 \times$ $10^{-5}$ ), "regulation of cell proliferation" (GO:0032944, p.adj. $<3.5 \times 10^{-3}$ ) and "cell adhesion" (GO:0007155, p.adj. $<7.7 \times 10^{-4}$ ) suggesting a reduced migratory potential of retinal microglia in the Irf8 $\mathrm{KO}$ mice compared with controls (Fig. 3B). Among the downregulated DEGs, we found numerous microglia signature genes essential for microglia homeostasis, including the spalt like transcription factor 1 (Sall1, $\log 2 \mathrm{FC}=-10.69,-\log 10 p$ = 24.57), allograft inflammatory factor 1 (Aif1 or Iba1, $\log 2 \mathrm{FC}=-2.73,-\log 10 p=35.04)$, purinergic receptor P2Y, G-Protein couple 12 (P2ry12, $\log 2 \mathrm{FC}=-2.50,-$ $\log 10 p=45.06)$ and transmembrane 119 (Tmem119, $\log 2 \mathrm{FC}=-2.13,-\log 10 p=24.57)($ Fig. 3A,C). In accordance with the RNA-seq data, we found a strong immunoreactivity for TMEM119 and P2RY12 in Irf8competent rMG cells, which was almost absent in Irf8deficient rMG. Conversely, we found an increased immunoreactivity for the mannose receptor (CD206, Mrc1) in Irf8-deficient rMG, consistent with and further supporting the RNA-seq results (Fig. 3D). In addition, flow cytometry analysis of homeostatic $\mathrm{CD} 45^{+} \mathrm{CD} 11 \mathrm{~b}^{+} \mathrm{rMG}$ confirmed our RNA-seq results showing a reduced but still detectable protein expression of $\mathrm{CX}_{3} \mathrm{CR} 1$ and $\mathrm{CD} 64$ in Irf8-deficient rMG compared with controls with a trend towards lower MERTK and higher F4/80 expression as reported before in bMG [44] (Fig. 3E).

Taken together these results strongly suggest that IRF8 plays a critical role in maintaining the distribution, morphology and homeostasis of retinal microglia and has a substantial impact on the transcriptional activity of rMG in the steady state.

\section{IRF8 is not required for the development of the retinal structure, vasculature and function}

Having established the quantitative and qualitative changes in Irf8-deficient rMG, we next investigated whether Irf8-deficiency influences retinal structure and electroretinal function during steady state. To this end, we examined the retina of adult Irf8-deficient and control mice using color fundus photography (CF), fluorescein angiography (FA), optical coherence tomography (OCT), electroretinography (ERG) and immunohistochemical staining of retinal flat mounts for retinal vessels (Suppl. Figure 3).

In general, Irf8 KO mice showed regular retinal structure, vasculature and function compared with controls. Qualitative assessment of retinal structure and vessels on CF and FA images of Irf8-deficient and control mice revealed no gross abnormalities, particularly no vascular dye leakage as an indicator of disturbed vascular architecture or spontaneous neovascularisation (Suppl. Figure 


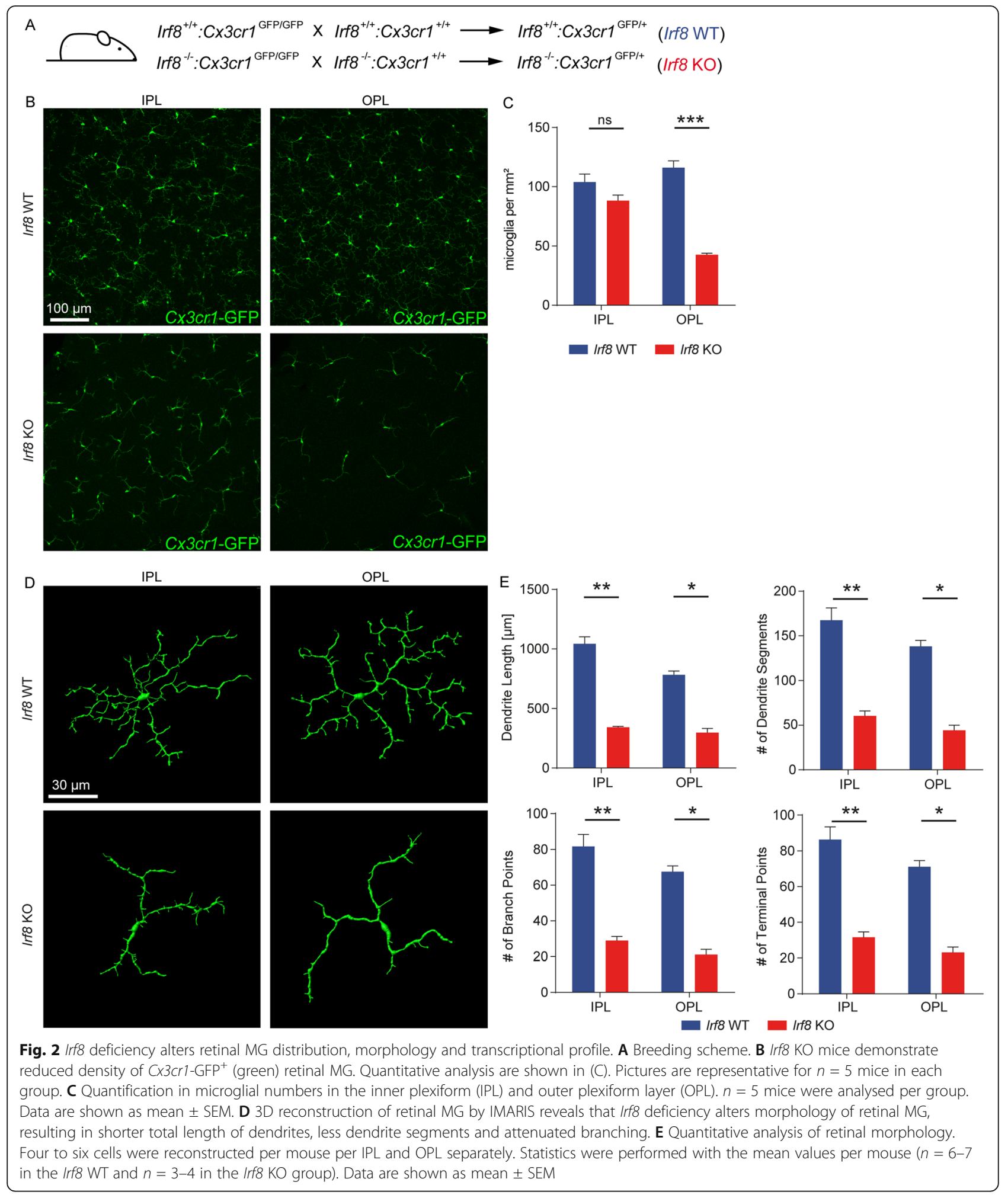

3A,B). In OCT images, the thickness of the inner nuclear layer (INL) and the outer nuclear layer (ONL) containing the photoreceptors (PR) was similar in Irf8-deficient mice (INL: $15.2 \pm 0.5 \mu \mathrm{m}$, ONL: $69.8 \pm 1.0 \mu \mathrm{m}$ ) compared with control animals (INL: $15.8 \pm 0.4 \mu \mathrm{m}$, ONL: $72.3 \pm 1.0 \mu \mathrm{m}$, Suppl. Figure 3C). ERG measurements demonstrated similar dark-adapted scotopic and light-adapted photopic responses in Irf8 $\mathrm{KO}$ mice and 


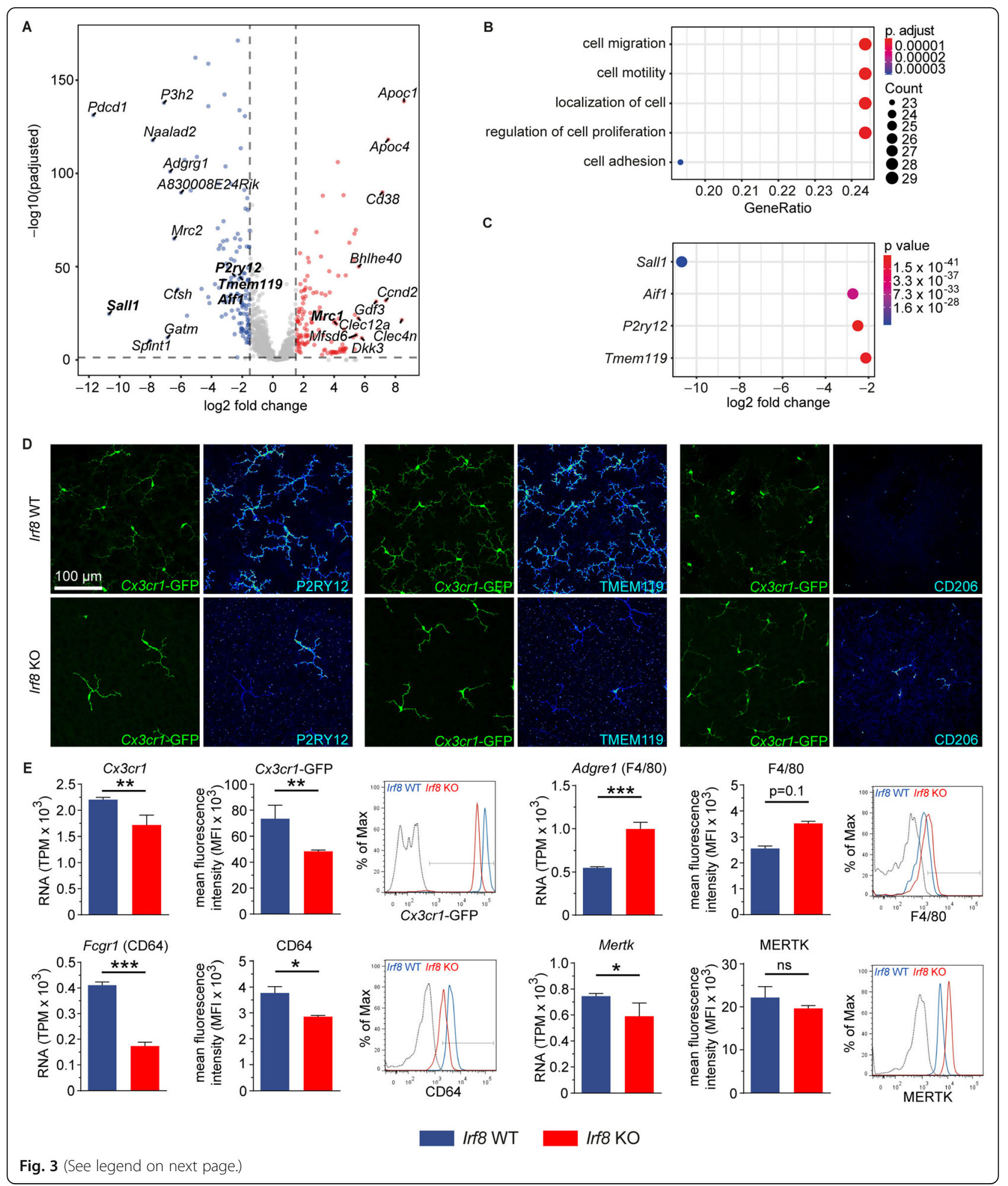


(See figure on previous page.)

Fig. 3 Irf8 deficiency leads to expression loss of homeostatic signature genes. A Volcano plot of differentially expressed genes in IIf8 KO retinal MG ( $n=3)$ compared with control $(n=5)$. Significantly up- and downregulated genes are shown in red and blue, respectively. The top significantly up-and downregulated genes are labelled. B The top 5 downregulated $\mathrm{GO}$ clusters in Irf8 KO retinal MG. Significance is represented as p.adjust, the size of each data circle indicates the number of genes involved in each enriched $\mathrm{GO}$ term. $\mathbf{C}$ Representative signature genes found to be highly expressed in competent retinal MG are significantly downregulated in the Irf8 KO mice. D Immunohistochemistry of retinal flat mounts demonstrate a strong immunoreactivity for P2RY12 and TMEM119 shown as colour-coded signal intensity in Iff-competent retinal MG that is reduced or absent in Iff8 KO mice. The mannose receptor CD206 (encoded by Mrc1) is absent under homeostatic conditions but detectable under Irf8-deficient conditions. E Myeloid expression levels shown as transcripts per million (TPM) and analogue surface marker expression, as determined by flow cytometry, of Iff8 WT (blue) and IIf8 KO (red) mice, expressed as mean fluorescence intensity (MFI) (left). Representative histograms are shown (right) including fluorescence minus one controls (grey line). Six mice per group were analysed for $\mathrm{CX}_{3} \mathrm{CR} 1, \mathrm{CD} 64$ and MERTK, three mice per group for F4/80. Data are shown as mean $\pm \mathrm{SEM}$

controls (Suppl. Figure 3D). Specifically, no significant difference was detected between Irf8 KO mice and controls with respect to scotopic a-waves emanating from rods, scotopic b-waves corresponding to depolarisation of bipolar cells, and photopic b-waves arising from cones. Immunohistochemical staining of retinal flat mounts revealed a regular retinal vasculature in $\operatorname{Irf} 8 \mathrm{KO}$ mice, including equal numbers of arteries labelled by smooth muscle actin (SMA, Irf8 WT $6.2 \pm 0.4$, Irf8 KO: $5.9 \pm 0.3$ per animal) and major vessels stained with Isolectin-B 4 (IB4, Irf8 WT: $11.3 \pm 0.5$, Irf8 KO: $11.0 \pm$ 0,5 per retinal per animal, Suppl. Figure 3E). Additionally, branch points in the central superficial vascular plexus (Irf8 WT: $17.0 \pm 2.7$, Irf8 KO $22.1 \pm 2.4$ per animal), central deep plexus (Irf8 WT: $8.7 \pm 4.7$, Irf8 KO: $74.0 \pm 3.4$ per animal), peripheral superficial plexus (Irf8 WT: $29.0 \pm 3.5$, Irf8 KO: $28.1 \pm 2.0$ per animal) and peripheral deep plexus (Irf8 WT: $59.0 \pm$ 5.1, Irf8 KO: $63.9 \pm$ 5.7 per animal) were similar between both groups (Suppl. Figure 3F).

Overall, these data show that $\operatorname{Irf} 8$ is not essential for the development and maintenance of homeostatic retinal structure, vascular network and function. This is particularly surprising given the significant changes in rMG cell numbers in the Irf8 $\mathrm{KO}$ mice during development and in the adult. Thus, the impaired retinal MG cell morphology and expression profile in otherwise unremarkable retinal homeostasis in Irf8-deficient mice provide a unique opportunity to investigate the role of retinal MG in the development of CNV.

\section{Irf8 deficiency aggravates CNV formation}

Retinal MG change their phenotype and transcriptional profile after tissue injury and modulate the development of pathological CNV, which represents a hallmark of neovascular AMD [59]. To investigate the role of IRF8 in microglial cell activation after tissue injury and formation of CNV, we next studied Irf8-deficient and control mice in the laser-induced CNV model. Both Irf8 KO and Irf8 WT mice developed typical laser-induced CNV 7 days after laser photocoagulation visible, as hyperfluorescent lesions with clear demarcation in FA images (Fig. 4A). Quantification of hyperfluorescent $\mathrm{CNV}$ areas in angiograms revealed more than 2-fold larger $\mathrm{CNV}$ lesions in Irf8 $\mathrm{KO}$ mice (8603 \pm 1309 pixels per animal) compared with controls $(3697 \pm 425$ pixels per animal, $p<0.005)$. Measurement of collagen type IV-labelled CNV area on RPE/ choroidal flat mounts confirmed significantly enlarged $\mathrm{CNV}$ lesions in Irf8 KO mice $\left(52143 \pm 7670 \mu \mathrm{m}^{2}\right)$ compared with Irf8 WT $\left(25203 \pm 4156 \mu \mathrm{m}^{2}, p<0.005\right.$, Fig. 4B). As expected, microscopic evaluation of RPE/choroidal flat mounts revealed that activated amoeboid Cx3cr1$\mathrm{GFP}^{+}$cells accumulate at CNV lesions in Irf8 $\mathrm{KO}$ mice as well as in controls. The number of $\mathrm{C} x 3 \mathrm{cr} 1-\mathrm{GFP}^{+}$cells around $\mathrm{CNV}$ lesions, however, was significantly decreased in Irf8 KO mice (34 \pm 7 cells per lesion per animal) compared with control animals (84.6 \pm 8.8 cells per lesion per animal, $p<0.001$, Fig. 4C). In addition, the number of rMG significantly increased in the IPL above $\mathrm{CNV}$ areas in controls, whereas no such increase was observed in Irf8 $\mathrm{KO}$ mice, further pointing to a defect in rMG migration (Suppl. Figure 4).

Taken together, Irf8-deficient mice revealed reduced MG cell numbers suggesting an impaired MG migratory behaviour under physiological and pathological conditions which was associated with increased $\mathrm{CNV}$ lesion size in the laser-CNV model.

\section{Retinal microglia rather than infiltrating monocytes account for the larger CNV lesions in the Irf8 KO mice}

Since $\mathrm{Cx} 3 \mathrm{cr} 1$ is expressed in retinal microglia and infiltrating monocyte-derived macrophages from the blood [26], the observed $C \times 3 c r 1^{\mathrm{GFP} /+}$ positive cells around CNV lesions could belong to both cell populations. However, Irf8-deficient mice are characterized by a low number of peripheral monocytes, which suggests that very few monocytes from the blood infiltrated the CNV lesion (Terry et al, 2015 PMID: 25277331). To investigate the influence of peripheral monocytes in our model, we next performed bone marrow transplantation experiments with bone marrow from CAG-RFP reporter animals to restore the peripheral monocyte pool in Irf8deficient mice with Irf8 potent monocytes (Fig. 5A). Following head-shielded bone marrow transplantation, we observed a successful reconstitution of $\mathrm{RFP}^{+}$Irf8-potent peripheral monocytes in Irf8-deficient animals 


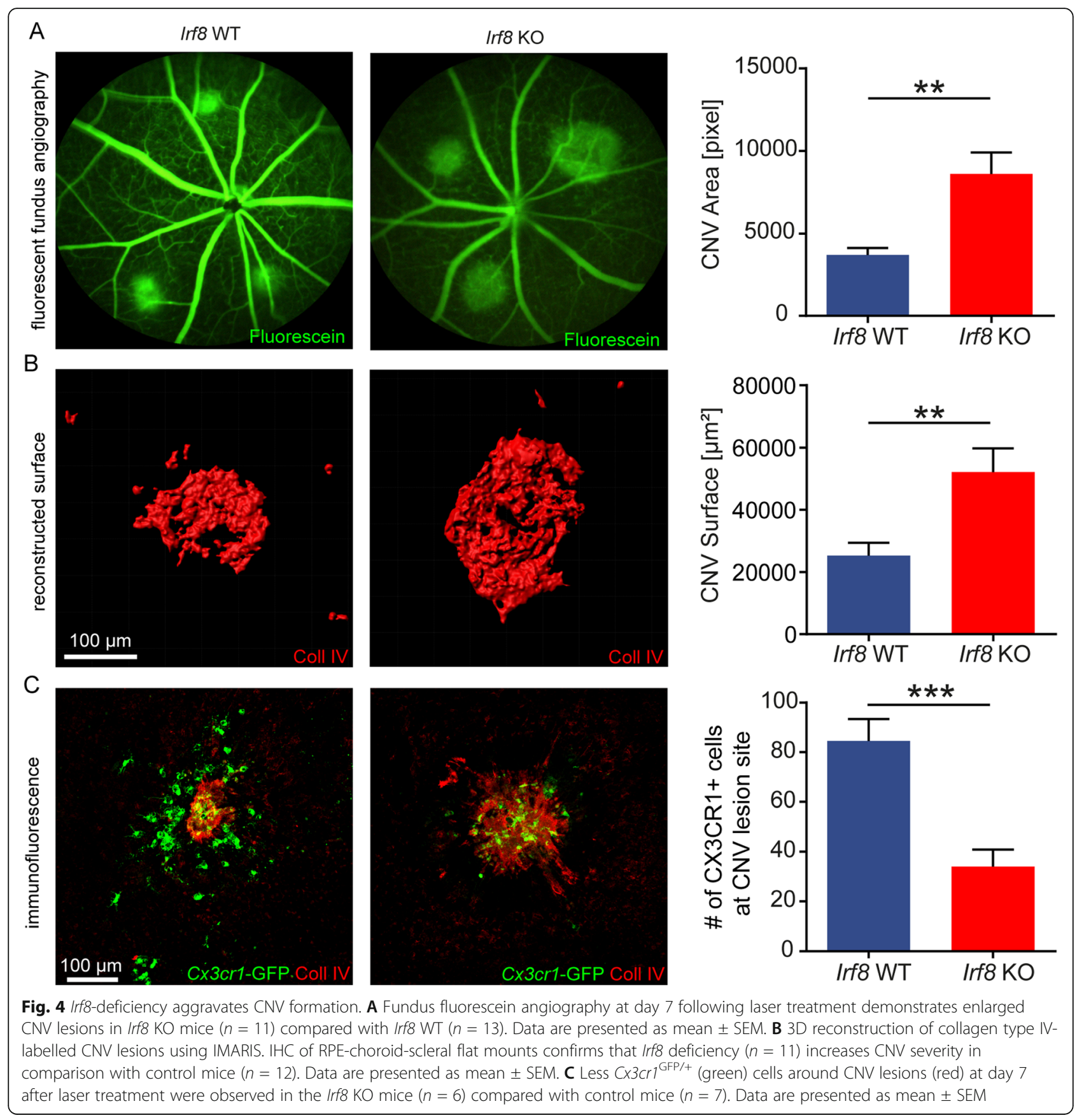

compared with controls by using flow cytometry (Irf8 KO: $95.78 \pm 0.59 \%\left(\right.$ RFP $\left.^{+} L y 6 C^{\text {hi }}\right), 97.42 \pm 0.14 \%$ $\left(\right.$ RFP $^{+}$Ly6C $\left.^{\text {lo }}\right)$, Irf8 WT: $31.19 \pm 4.85 \%\left(\right.$ RFP $^{+}$Ly6C $\left.^{\text {hi }}\right)$, $40.24 \pm 5.5 \%\left(\right.$ RFP $^{+}$Ly6C $\left.C^{\text {lo }}\right)$, Fig. 5B). The observed higher recombination efficiency of Ly6C $C^{\text {hi }}$ and $L y 6 C^{\text {lo }}$ monocytes in $\operatorname{Irf} 8 \mathrm{KO}$ is likely due to the initially low abundance of these cells and their respective progenitors in Irf8 $\mathrm{KO}$ mice. Of note, due to the head shielding, the recipients' bone marrow in the skull is still active and not substituted by the donor cells that could explain the comparably lower recombination efficiency in Irf8 WT mice (Mildner et al, 2007 PMID: 18026096). Interestingly, the reconstituted Irf8-deficient animals still demonstrated increased CNV lesion size that was approximately twice as large compared with reconstituted Irf8 WT animals (Irf8 KO 82,009 $\pm 16,242 \mu \mathrm{m}^{2}$; Irf8 WT $\left.53,386 \pm 4793 \mu \mathrm{m}^{2}, p=0.06\right)$ which was associated with slightly reduced numbers of $\mathrm{RFP}^{-} \mathrm{GFP}^{+}$microglia at sites of CNV (Irf8 KO 74 \pm 16.5 ; Irf8 WT $197.3 \pm$ 12.1, $p=0.14$ ) (Fig. 5C,D). The numbers of reconstituted $\mathrm{RFP}^{+} \mathrm{GFP}^{-}$monocyte-derived macrophages, in contrast, were around twofold increased in Irf8-deficient mice 


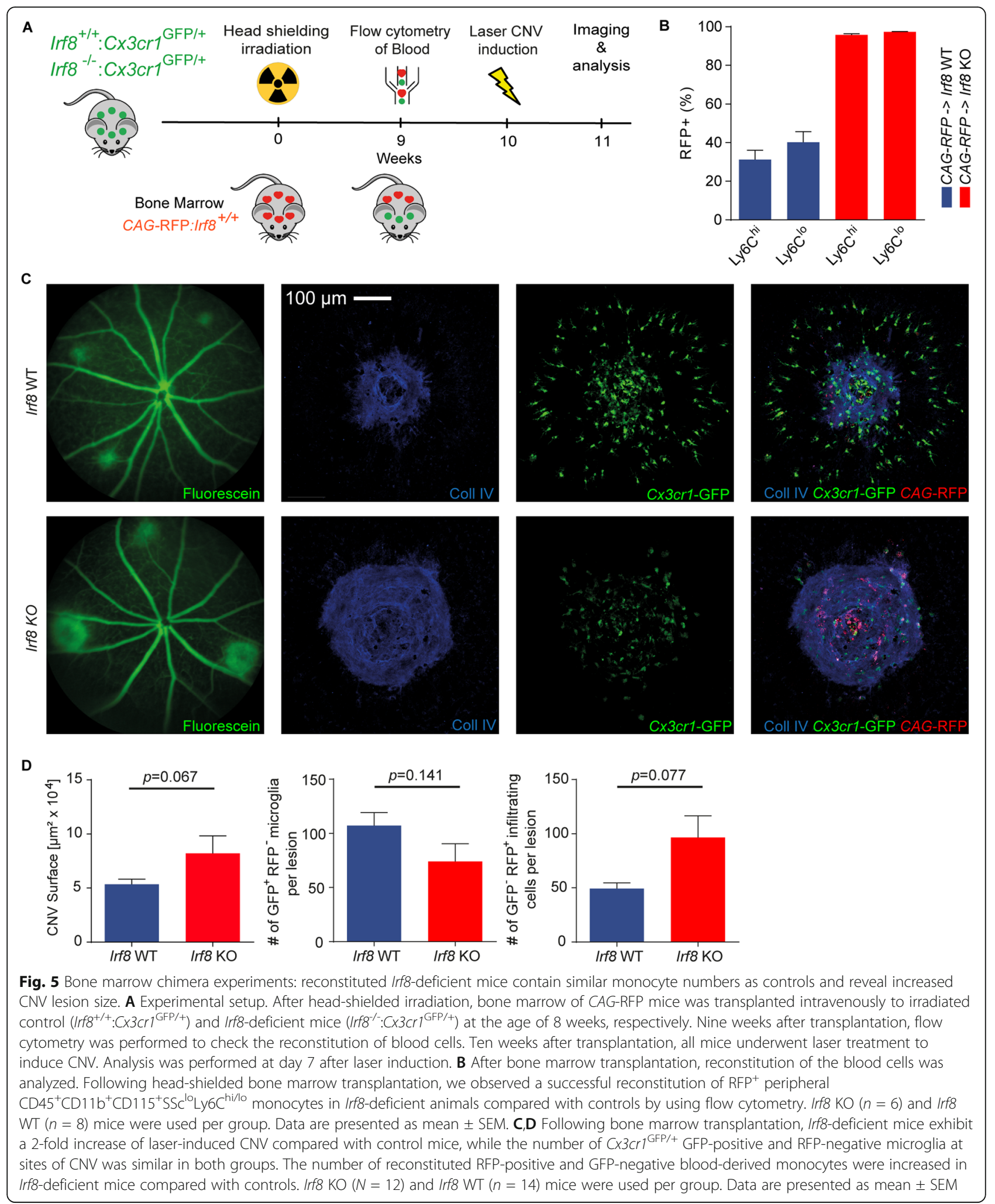




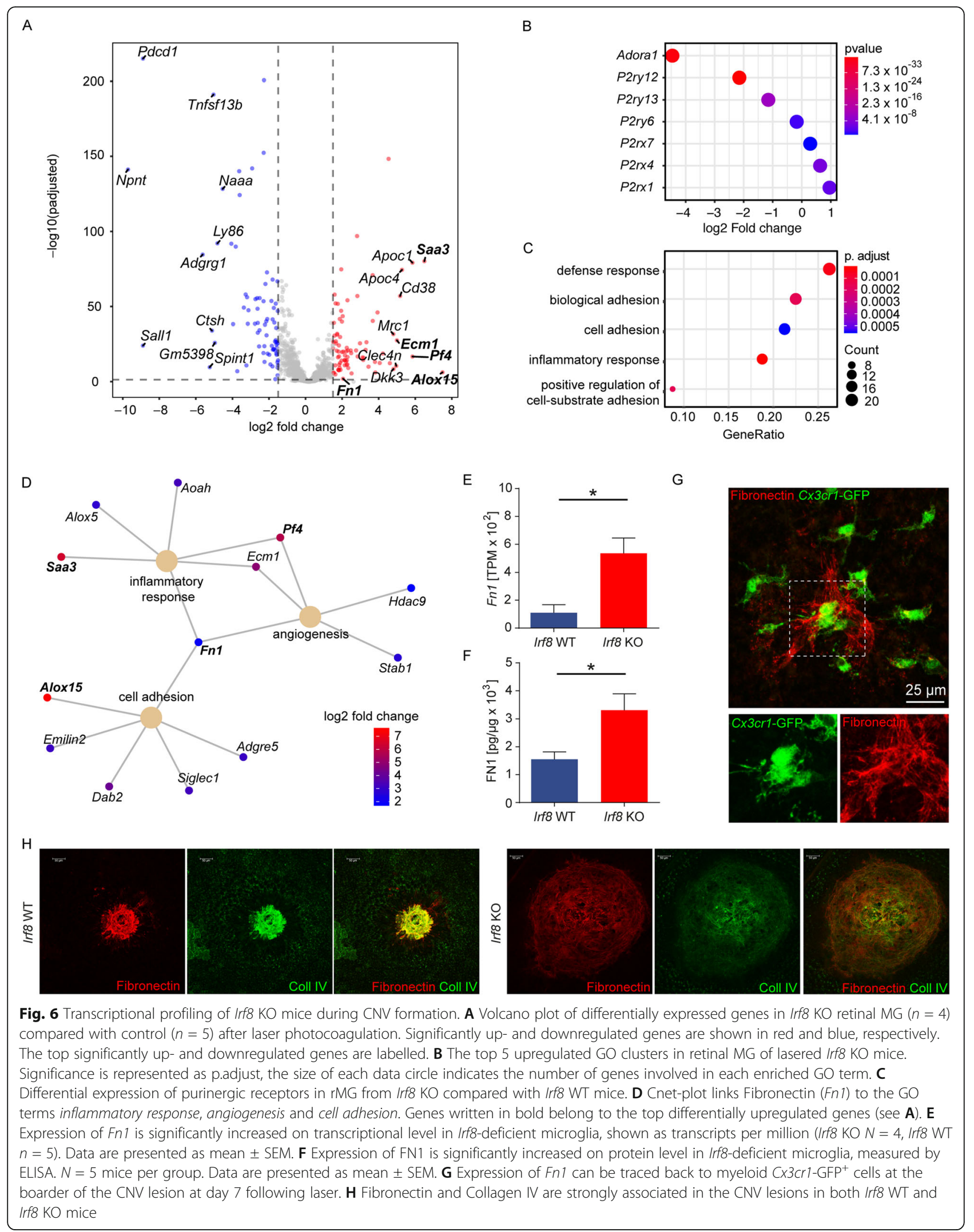


compared with controls (Irf8 KO $96.6 \pm 20.1$; Irf8 WT $49.25 \pm 5.3, p=0.07$ ) (Fig. 5D).

\section{Transcriptional profile of Irf8 KO retinal microglia during CNV formation}

In order to decipher the molecular mediators of enhanced $\mathrm{CNV}$ formation in Irf8 $\mathrm{KO}$ mice, we next isolated $\mathrm{CNV}$-associated rMG from Irf8 $\mathrm{KO}$ and control mice by flow cytometry and analysed the cells using RNA-seq. In total, we found 84 genes that were differentially upregulated and 78 genes that were downregulated in Irf8-deficient microglia 7 days following laser injury. In line with the RNA-seq analysis under homeostatic conditions, we identified similar DEG that were downor upregulated in Irf8-deficient microglia after tissue injury, such as Sall1, P2yr12 and Mrc1 (Fig. 6A). The downregulation of P2ry12 in laser-treated Irf8 $\mathrm{KO}$ mice prompted us to explore the expression of other purinergic receptors which are critical for MG cell activation and migration. Here, we found several other genes encoding purinergic receptors to be strongly downregulated in Irf8-deficient MG, such as Adora1, P2ry12 and P2ry13, underlining the proposed migration defect upon laser injury (Fig. 6B). Furthermore, we analysed the expression of key M1 (Cd86, H2-Ab1, Tlr2) and M2 signature genes $(C D 163, M r c 1)$ in isolated $\mathrm{CNV}$-associated MG in Irf8-deficient mice and control animals (Suppl. Figure 5). We found that common M1 markers such as Cd86, $H 2-A b 1$ and Tlr2 were significantly downregulated in Irf8-deficient MG compared with Irf8-potent MG in the laser CNV model. On the other hand, some of the common M2 markers, such as Cd163 and Mrc1, were significantly upregulated in Irf8-deficient retinal MG compared with Irf8-potent MG at sites of CNV suggesting a M1 to M2 polarization in Irf8-deficient MG compared with wild-type MG in the laser CNV model.

Next, we analysed the DEG that were upregulated in Irf8-deficient MG by GO cluster analysis and found an activation of biological processes such as "defense response" (GO:0006952, p.adj. < 8.4 × 10 $0^{-6}$ ), "biological adhesion" (GO:0022610, p.adj. $\left.<2.9 \times 10^{-4}\right)$, "cell adhesion" (GO:0007155, p.adj. < $\left.6.1 \times 10^{-4}\right)$, "inflammatory response" (GO:0006954, p.adj. < $9.5 \times 10^{-6}$ ) and "positive regulation of cell substrate adhesion" (GO:0010811, p.adj. $<1.9 \times 10^{-3}$ ) in Irf8-deficient MG (Fig. 6C). A closer look at the DEG of three key GO clusters essential for CNV development, namely "angiogenesis", "inflammatory response" and "cell adhesion", revealed Fibronectin, a profibrotic mediator encoded by $F n 1$, as a prominent upregulated gene in Irf8-deficient MG linking all three biological processes (Fig. 6D). In addition, we found a marked increase in the expression of other profibrotic factors such as Fgf2 and Spp1 and a subtle increase in the expression of Tgfb1 in $\mathrm{CNV}$-associated retinal MG in Irf8-deficient mice compared with control mice (data not shown). Consistent with the increased number of $F n 1$ transcripts in Irf8-deficient MG, we found significantly increased FN1 protein levels in the $\mathrm{RPE} /$ choroid of Irf8-deficient mice $(3314 \pm 587.1 \mathrm{pg} / \mu \mathrm{g}$ protein) compared with controls $(1550 \pm 268.5 \mathrm{pg} / \mu \mathrm{g}$ protein, $p<0.05$ ) using ELISA on tissue lysates (Fig. 6E, F). Immunohistochemical studies showed that FN1 expression was restricted to the area of $\mathrm{CNV}$ lesions and expressed by microglia at sites of CNV (Fig. 6G). In addition to the enlarged collagen IV-positive CNV lesion described above, Irf8-deficient mice showed a markedly enlarged FN1-positive CNV lesion compared with WT mice, indicating increased fibrosis (Fig. 6H).

Taken together, these studies show that Irf8-deficient microglia exhibit a significantly altered expression profile in the laser $\mathrm{CNV}$ model with downregulated migratory genes, such as purinergic receptors, and upregulated pro-fibrotic factors, such as $F n 1$.

\section{Discussion}

The interferon regulatory factor 8 (IRF8) is an essential transcription factor for the development, maturation and homeostasis of microglia (MG) in the brain and other tissue macrophages $[19,21,27]$. However, the role of IRF8 for retinal MG (rMG) during homeostasis and neovascular eye disease has not been elucidated so far. In this study, we show that Irf8 is essential for a mature rMG gene expression profile and influences MG morphology, migration and the response to pathological neovascularisation.

Our results show that Irf8 is strongly expressed in rMG in the steady state compared with other IRF family members. The expression of Irf8 in rMG was even substantially higher than in brain MG (bMG), suggesting a distinct and tissue-specific function of Irf8 in microglia of the retina. Morphological analysis revealed that Irf8deficient mice exhibited an overall reduced branching of rMG as well as a decreased MG cell number specifically in the outer plexiform layer, which was already observed during postnatal development. These findings recapitulate findings in the brain $[21,27,39,44]$ and point to a migratory defect that is already present during postnatal retinal layering and maintained into adulthood. To gain further insight into the molecular changes in Irf8-deficient rMG, we isolated rMG by flow cytometry and analysed their transcriptional profile by RNA sequencing. Our analysis revealed significant transcriptional differences between Irf8-potent and Irf8-deficient microglia, which is consistent with several in vitro studies $[25,40]$. We found that Sall1 was the most downregulated MG signature gene in the retina of Irf8-deficient mice. This is of particular functional importance since SALL1 is a critical transcription factor for maintaining the 
homeostatic gene expression pattern of MG and thus regulates, for example, the expression of MG signature genes such as Aif1, P2ry12 and Tmem119 [6, 33, 55]. Accordingly, we found Tmem119 and P2ry12 as significantly reduced in Irf8-deficient rMG recapitulating the findings from human brain microglia which suggest a dependence of P2RY12 expression on IRF8 signalling [5]. Notably, because SALL1 is not exclusively expressed by microglia but also by astrocytes and oligodendrocytes $[8,38,48]$, non-cell autonomous effects of this gene cannot be excluded. Further, single-cell RNA-seq (scRNASeq) of conditional Irf8 KO mice $\left(F c g r 1-C r e: I r f 8^{\mathrm{fl} / \mathrm{fl}}\right)$ revealed a strong downregulation of Sall1, Aif1, Tmem119 and P2ry12 in bMG while other genes, typically expressed in macrophages, like $M r c 1$, encoding the mannose receptor CD206, were upregulated [58]. In line with this study, we found a strong upregulation of Mrc1 on transcriptional level and the encoded mannose receptor CD206 on protein level in Irf8-deficient rMG which is consistent with data from Irf8-deficient bMG [44]. In addition, other myeloid genes like Fcgrl, also known as CD64, were downregulated on RNA and protein level. These findings strongly support common transcriptional changes between retinal MG and brain MG under Irf8deficient conditions, as shown before in a direct comparison of wild-type rMG and bMG by scRNA-seq [59]. Furthermore, it underscores that retinal microglial differentiation and maturation are highly dependent on a defined transcriptional program instructed by PU.1 [16], SALL1 [29] and IRF8.

To identify functionally related gene signatures, we performed a gene ontology (GO) enrichment analysis that revealed a downregulation of the clusters "cell migration", "cell motility", "cell adhesion" and "regulation of cell proliferation" in adult Irf8-deficient rMG in the steady state. The downregulation of these GO terms was consistent with the reduced number of rMG in the OPL in adult Irf8 $\mathrm{KO}$ mice, supporting previous in vitro data describing IRF8 as an essential transcription factor for microglial motility and migration [28, 39]. The significant downregulation of genes relevant for cell migration could also explain the observed developmental phenotype and point to an impaired Irf8-dependent perception of guiding cues. During development, neurons constantly release guiding cues, such as purines and other extracellular nucleotides which guide microglia to colonise the developing outer retina $[1,37]$. Irf8-dependent downregulation of sensors that detect these guiding cues, such as Adora1 or P2yr12, may be responsible for the disruption of purinergic signalling in Irf8-deficient rMG, which attenuates their migratory capacity. Indeed, in vitro cultivated brain MG lacking Irf8 had a strongly reduced phosphorylation of AKT after ATP treatment, thereby diminishing the ATP-mediated signalling pathways of P2RY12 [40]. This hypothesis is supported by in vitro studies showing a diminished entry of rMG into retinal explant cultures following an interruption of purinergic signalling [37]. Furthermore, proliferation of myeloid cells relies on IRF8 signalling [61] which could explain the insufficient compensatory expansion of MG in the OPL and contribute to the niche-dependent phenotype with reduced microglial density especially in the OPL in adult Irf8-deficient mice.

Despite the aforementioned significant changes in MG cell density and transcriptional profile, Irf8-deficient mice exhibited a normal retinal structure, vascular supply, and physiological function. This is particularly surprising as retinal MG are in close contact with retinal vessels, especially during development, and are known to shape the mature retinal vasculature $[9,13,16,18]$. This suggests that Irf8-deficient MG are functionally sufficient to accompany physiological retinal development and that minor developmental disturbances, which we cannot completely rule out, can be compensated over time. The fact that Irf8-deficient microglia exhibit transcriptional changes that reduce their responsiveness while maintaining a normal retinal phenotype, provides an ideal setting to study the role of microglia in the development of choroidal neovascularisation, which is known to be associated with significant microglia activation and migration $[35,52,59]$.

In the laser-induced $\mathrm{CNV}$ model, which mimics aspects of neovascular AMD, we found significantly increased CNV lesion size under Irf8-deficient conditions. At the same time, the overall numbers of Irf8-deficient Cx3cr1-GFP ${ }^{+}$cells were significantly reduced around the lesions compared with wild type. In line with this finding, MG density in the IPL above CNV lesions was significantly decreased in Irf8 $\mathrm{KO}$ mice compared with wild type. These observations may be mediated either by a migration defect caused by impaired purinergic signalling [45] or by reduced microglial proliferation of Irf8deficient MG, or both, as discussed above. Interestingly, the present study shows that impaired migration and a lower number of MG in the vicinity of $\mathrm{CNV}$ are associated with a more severe $\mathrm{CNV}$ phenotype in Irf8-deficient mice. This finding is in contrast to previous studies showing that depletion of retinal or circulating myeloid cells by clodronate is associated with reduced CNV size suggesting an anti-angiogenic effect of myeloid cells on CNV $[14,34,51]$. On the other hand, however, our results are consistent with reports showing that increased accumulation of myeloid cells in neovascular lesions is associated with decreased $\mathrm{CNV}$ and that myeloid cells use FasL (CD95L) to inhibit CNV formation [2]. These seemingly contradictory contributions of myeloid cells to $\mathrm{CNV}$ development could be reconciled by the potentially different roles of resident MG and infiltrating 
monocyte-derived macrophages. To investigate the distinct roles of these cellular populations, this study exploits Irf8-deficient mice which are characterized by a near absence of circulating monocytes and, at the same time, altered but present resident retinal microglia. The enlarged $\mathrm{CNV}$ lesions in Irf8-deficient mice associated with reduced numbers of $\mathrm{C} x 3 \mathrm{crl}-\mathrm{GFP}^{+}$resident microglia cells may thus be interpreted as a consequence of dysfunctional pro-fibrotic Irf8-deficient MG or as a result of insufficient numbers of CNV-suppressing microglia in Irf8-deficient animals. The latter may in turn indicate a general protective role of wild-type microglia which would be in line with previous studies $[2,46]$. The hypothesis that deficient microglia substantially influence CNV size in Irf8-deficient mice is further supported by the head-shielded bone marrow transplantation experiments performed in this study, which showed enlarged $\mathrm{CNV}$ in Irf8-deficient animals compared with controls despite successful reconstitution of Irf8-potent peripheral monocytes. However, we cannot exclude the possibility that other Irf8-expressing cell types in the retina or from the blood contribute to the increased $\mathrm{CNV}$ lesion size in Irf8-deficient mice. Since Irf8 expression in the retina was mainly restricted to retinal microglial cells, which represent the most numerous myeloid cell population in $\mathrm{CNV}$, in contrast to a low number of infiltrating peripheral monocytes [59], we consider this possibility rather unlikely. Furthermore, heterozygous Cx3cr1 $1^{G F P /+}$ expression could have an impact on myeloid cells and be associated with an inflammatory phenotype. However, since both Irf8 wild-type and knockout mice in our study were consistently heterozygous for $C \times 3 c r 1$, we consider this effect negligible and used the $\mathrm{C} \times 3 \mathrm{cr} 1^{\mathrm{GFP} /+}$ line to visualise $\operatorname{Irf} 8$ wild-type and knockout mice in a comparable manner, since other standard markers such as IBA1 are dysregulated and cannot be used [44].

To explore potential mechanisms in Irf8-deficient retinal MG contributing to $\mathrm{CNV}$ formation, we next performed RNA-sequencing on sorted retinal MG at sites of $\mathrm{CNV}$.

Among others, we found that purinergic receptors, encoded by genes such as P2ry12, P2ry13 and Adora1, were significantly downregulated in Irf8 KO rMG after laser injury. Since these receptors are critical for the recognition of ATP released during tissue injury and thus control cell migration, their downregulation may be at least partly responsible for the reduced MG cell number at sites of CNV in Irf8-deficient mice [12, 23, 30]. This hypothesis is in line with in vitro work by Masuda et al. showing a downregulation of genes encoding purinergic receptors, including P2ry12 and P2rx4, in Irf8-deficient brain microglia which was associated with a migration defect in vitro [39]. It is interesting to note that Irf8- deficient retinal microglia accumulating at $\mathrm{CNV}$ showed less M1 and more M2 signature gene expression compared with wild-type MG at $\mathrm{CNV}$, suggesting increased M1 to M2 polarization in Irf8-deficient MG. This is of particular clinical interest as some studies have suggested that more M2-like macrophages, which are assumed to be pro-angiogenic, accumulate at the site of wet compared with dry AMD and that the pathological shift of macrophage polarization may contribute to the pathogenesis of CNV in neovascular AMD [7]. The expression signatures or myeloid cells at the lesion site are likely to be more complex than the aforementioned polarization state; however, it confirms an altered signature of a set of well-known and characterized markers. Furthermore, GO cluster analysis showed that Irf8-deficient rMG exhibit increased activity of biological processes that are critical for inflammation and cell adhesion, which points to an interaction of microglia with extracellular matrix components driving $\mathrm{CNV}$ formation. We hereby identified Fibronectin, encoded by the $F n 1$ gene, as being significantly higher expressed in Irf8 KO rMG and linking the GO terms "cell adhesion", "inflammatory response" and "angiogenesis". The immunohistochemical and ELISA analyses confirmed that the expression of Fibronectin protein was also significantly increased at sites of $\mathrm{CNV}$, suggesting that Irf8-deficient MG or other cells affected by the loss of Irf 8 contribute to increased Fibronectin abundance at sites of CNV. Of note, collagen IV fibers were reported to depend on an established Fibronectin matrix directly co-localizing with Fibronectin fibers in vitro that could be functionally related to our observation of increased $\mathrm{CNV}$ lesion size promoted by higher Fibronectin expression in rMG [42, 43]. Furthermore, we found a marked increase in the expression of the pro-fibrotic factors $F g f 2$ and Spp1 and a subtle increase in the expression of $T g f b 1$ in $\mathrm{CNV}$-associated retinal $\mathrm{MG}$ in Irf8-deficient mice compared with control mice. Since FGF2 and SPP1 are important mediators of scarring and have been identified in human choroidal neovascular membranes $[17,52]$, overexpression of these factors in retinal MG may have further contributed to increased $\mathrm{CNV}$ lesion size in Irf8-deficient mice. This is of particular interest as pharmaceutical inhibition of SPP1 has been shown to modulate $\mathrm{CNV}$ formation and inhibition of FGF2 was associated with reduced CNV and subretinal fibrosis in a laser-induced mouse CNV model [3, $41,52]$.

\section{Conclusions}

In conclusion, this study identifies IRF8 as a critical mediator for the morphology, distribution and expression profile of retinal microglia, and for transformation to a reactive phenotype. The niche-dependent phenotype 
already present during postnatal development, the altered morphology and the disturbed rMG distribution did not lead to any impairment of retinal morphology and function in the steady state, but to enlarged lesions in the laser $\mathrm{CNV}$ model. This highlights the importance of IRF8 and retinal MG for the development of pathological neovascularisation in the eye and highlights the potential of immunomodulatory therapeutic interventions of rMG recruitment in retinal disease.

\begin{abstract}
Abbeviations
Adora1: Adenosine A1 receptor; Aif1: Allograft inflammatory factor 1; AMD: Age-related macular degeneration; ATP: Adenosine triphosphate; CNS: Central nervous system; CNV: Choroidal neovascularization; CSF1R: Colony stimulating factor 1 receptor; ELISA: Enzyme-linked immunosorbent assay; ERG: Electroretinography; FFA: Fundus fluorescein angiography; FN: Fibronectin; GO: Gene ontology; INL: Inner nuclear layer; IRF: Interferon regulatory factor; MG: Microglia; OCT: Optical coherence tomography; ONL: Outer nuclear layer; P2ry12: Purinergic receptor P2Y, GProtein couple 12; PR: Photoreceptors; Sall1: Spalt-like transcription factor 1; Tmem119: Transmembrane 119; WT: Wild type
\end{abstract}

\section{Supplementary Information}

The online version contains supplementary material available at https://doi. org/10.1186/s12974-021-02230-y.

Additional file 1. Supplemental figure 1 Irf8 is expressed predominantly in retinal MG and in some bipolar or Müller cells. No Irf8-VENUS expression could be detected co-localised with GFAP (A), Blll-Tubulin (B) and Collagen IV (E), indicating that IRF8 is not expressed in retinal astrocytes, ganglion cells or vessels. All IBA ${ }^{+}$cells exhibited a strong Irf8VENUS signal (C) suggesting that all retinal MG express IRF8. Some $\mathrm{CHX} 10^{+}$cells could be co-localised with Irf8-VENUS expression (D) demonstrating that some bipolar cells or Müller cells express VENUS.

Additional file 2. Supplemental figure 2 Temporal and spatial distribution of retinal microglia during development. A Representative pictures showing the numbers of microglia per field of view in Irf8 WT and Irf8 $\mathrm{KO}$ mice in comparison between the ganglion cell and inner plexiform layer (GCL/PL) and the developing neuroblast layer (NBL) or outer plexiform layer (OPL), respectively, at postnatal day (P) 1, P7 and in adult mice. B Quantification thereof. Data are presented as mean \pm SEM.

Additional file 3. Supplemental figure 3 Irf8 deficiency does not affect the retinal structure, function and vasculature. A-C Representative color fundus images (A), fluorescein angiography (B) and optical coherence tomography (OCT) images of Iff8 WT and Irf8 KO mice. C) Both Iff8 WT (blue, $\mathrm{n}=12$ ) and Irf8 $\mathrm{KO}(\mathrm{red}, \mathrm{n}=12$ ) mice displayed a regular retinal structure, a similar thickness of the inner nuclear layer and the outer retina at 100 and $200 \mu \mathrm{m}$ from the optic nerve head in the optical coherence tomographs. Data are shown as mean \pm SEM. ONH = Optic nerve head. D Electroretinography (ERG). No significant difference was found between the Irf8 WT (blue, n=9) and Irf8 KO (red, n=9) mice concerning the darkadapted scotopic and light-adapted photopic ERG measurements at different flash intensities. Data are shown as mean \pm SEM. E Staining against smooth muscle actin (SMA, red) reveals a comparable number of arteries (Irf8 WT ( $n=5)$; Irf8 KO $(n=7)$ ) and major vessels (IIf8 WT ( $n=11)$; Irf8 KO ( $n=$ 13)) between both groups. Data are shown as mean \pm SEM. F No significant differences in vessel branch formation in the superficial (upper panel) or deep plexus (lower panel) in the central or peripheral area of the retina could be observed, compared between IIf8 WT $(n=6)$ and Irf8 $\mathrm{KO}(\mathrm{n}=7)$. Data are presented as mean \pm SEM.

Additional file 4. Supplemental figure 4 Spatial distribution of retinal microglia in CNV lesions. A Spatial distribution of microglia in the inner plexiform layer (IPL) in lasered and unlasered Irf8 WT and Irf8 KO mice. B Quantification of the numbers of microglia per field of view in the inner plexiform layer in lasered and unlasered Irf8 WT and IIf8 KO mice. Data are presented as mean \pm SEM.
Additional file 5. Supplemental figure 5: Polarisation markers expressed by CNV-associated microglia. The M1 and M2 polarisation markers Cd86, H2-Ab1, Trr2, Cd163 and Mrc1 (CD206) are shown as transcripts per million in comparison between Iff8 WT and KO under CNV conditions.

Additional file 6. Supplemental table 1: List of primer sequences.

Additional file 7. Supplemental table 2: List of antibodies used for immunohistochemistry and flow cytometry.

\section{Acknowledgements}

We would like to thank Gabriele Prinz, Michael Bach and Daniel Böhringer for excellent technical and bioinformatical assistance. We are grateful to Frank Rosenbauer for providing Irf8-VENUS reporter mice.

\section{Authors' contributions}

$\mathrm{PZ}, \mathrm{AS}, \mathrm{SB}, J \mathrm{JK}, \mathrm{FL}, \mathrm{YL}, J \mathrm{~J}, \mathrm{FL}, \mathrm{MB}, \mathrm{AT}, \mathrm{CH}$ and PW performed experiments and analysed data. JW analysed RNA-seq data. HA, GS, IH, KK and MP provided scientific input. CL and PW supervised the project and wrote the manuscript. The authors read and approved the final manuscript.

\section{Funding}

KK was supported by a project grant of the Fritz-Thyssen Foundation. $C L, I H$, KK and MP were supported by the SFB/TRR167. Open Access funding enabled and organized by Projekt DEAL.

\section{Availability of data and materials}

RNA sequencing data are available under the GSE accession number GSE160845 and GSE182504.

\section{Declarations}

\section{Ethics approval and consent to participate}

All animal experiments were authorized by the local animal care and use committee under the respective EU, national, federal and institutional regulations for animal experiments.

\section{Consent for publication}

All authors consented to publish this article.

\section{Competing interests}

The authors declare no conflict of interest in this study.

\section{Author details}

${ }^{1}$ Medical Faculty, Eye Center, University Hospital, University of Freiburg, Freiburg im Breisgau, Germany. ${ }^{2}$ Institute of Anatomy, Wuerzburg University, Wuerzburg, Germany. ${ }^{3}$ Cardiology and Angiology, University Heart Center, University of Freiburg, Freiburg im Breisgau, Germany. ${ }^{4}$ Medical Center and Faculty of Medicine, Institute for Experimental Cardiovascular Medicine, University Heart Center Freiburg Bad Krozingen, University of Freiburg, Freiburg, Germany. ${ }^{5}$ Medical Faculty, Institute of Neuropathology, University Hospital, University of Freiburg, Freiburg im Breisgau, Germany. ${ }^{6}$ CIBSS-Centre for Integrative Biological Signalling Studies, University of Freiburg, Freiburg, Germany. ${ }^{7}$ Medical Faculty, Center for Basics in NeuroModulation (NeuroModulBasics), University of Freiburg, Freiburg im Breisgau, Germany. ${ }^{8}$ Signalling Research Centres BIOSS and CIBSS, University of Freiburg, Freiburg im Breisgau, Germany. ${ }^{9}$ Institute of Anatomy, Leipzig University, Leipzig, Germany.

Received: 31 March 2021 Accepted: 4 August 2021 Published online: 20 September 2021

\section{References}

1. Akhtar-Schäfer I, Wang $L$, Krohne TU, Xu H, Langmann T. Modulation of three key innate immune pathways for the most common retinal degenerative diseases. EMBO Mol Med. 2018;10(10). https://doi.org/10.152 52/emmm.201708259.

2. Apte RS, Richter J, Herndon J, Ferguson TA. Macrophages inhibit neovascularization in a murine model of age-related macular degeneration. PLoS Med. 2006;3(8):e310. https://doi.org/10.1371/journal.pmed.0030310.

3. Beguier F, Housset M, Roubeix C, Augustin S, Zagar Y, Nous C, et al. The $10 q 26$ risk haplotype of age-related macular degeneration aggravates 
subretinal inflammation by impairing monocyte elimination. Immunity. 2020;53:429-441.e8.

4. Boeck M, Thien A, Wolf J, Hagemeyer N, Laich Y, Yusuf D, et al. Temporospatial distribution and transcriptional profile of retinal microglia in the oxygen-induced retinopathy mouse model. Glia. 2020;68(9):1859-73. https://doi.org/10.1002/glia.23810.

5. Böttcher C, Schlickeiser S, Sneeboer MAM, Kunkel D, Knop A, Paza E, et al. Human microglia regional heterogeneity and phenotypes determined by multiplexed single-cell mass cytometry. Nat Neurosci. 2019;22(1):78-90. https://doi.org/10.1038/s41593-018-0290-2.

6. Buttgereit A, Lelios I, Yu X, Vrohlings M, Krakoski NR, Gautier EL, et al. Sall1 is a transcriptional regulator defining microglia identity and function. Nat Immunol. 2016;17(12):1397-406. https://doi.org/10.1038/ni.3585.

7. Cao X, Shen D, Patel MM, Tuo J, Johnson TM, Olsen TW, et al. Macrophage polarization in the maculae of age-related macular degeneration: a pilot study. Pathol Int. 2011;61(9):528-35. https://doi.org/10.1111/j.1440-1827.2011. 02695.x.

8. Chappell-Maor L, Kolesnikov M, Kim J-S, Shemer A, Haimon Z, Grozovski J, et al. Comparative analysis of CreER transgenic mice for the study of brain macrophages: a case study. Eur J Immunol. 2020;50(3):353-62. https://doi. org/10.1002/eji.201948342.

9. Checchin D, Sennlaub F, Levavasseur E, Leduc M, Chemtob S. Potential role of microglia in retinal blood vessel formation. Invest Ophthalmol Vis Sci. 2006;47(8):3595-602. https://doi.org/10.1167/iovs.05-1522.

10. Colijn JM, Buitendijk GHS, Prokofyeva E, Alves D, Cachulo ML, Khawaja AP, et al. Prevalence of age-related macular degeneration in Europe: the past and the future. Ophthalmology. 2017;124(12):1753-63. https://doi.org/10.101 6/j.ophtha.2017.05.035

11. Combadiere C, Feumi C, Raoul W, Keller N, Rodero M, Pezard A, et al. CX3CR1-dependent subretinal microglia cell accumulation is associated with cardinal features of age-related macular degeneration. J Clin Invest. 2007; 117:2920-8.

12. Davalos D, Grutzendler J, Yang G, Kim JV, Zuo Y, Jung S, et al. ATP mediates rapid microglial response to local brain injury in vivo. Nat Neurosci. 2005; 8(6):752-8. https://doi.org/10.1038/nn1472.

13. Dudiki T, Meller J, Mahajan G, Liu H, Zhevlakova I, Stefl S, et al. Microglia control vascular architecture via a TGF $\beta 1$ dependent paracrine mechanism linked to tissue mechanics. Nat Commun. 2020;11:1-16.

14. Espinosa-Heidmann DG, Suner IJ, Hernandez EP, Monroy D, Csaky KG, Cousins SW. Macrophage depletion diminishes lesion size and severity in experimental choroidal neovascularization. Invest Ophthalmol VisSci. 2003; 44(8):3586-92. https://doi.org/10.1167/iovs.03-0038.

15. Fantin A, Lampropoulou A, Senatore V, Brash JT, Prahst C, Lange CA, et al. VEGF165-induced vascular permeability requires NRP1 for ABL-mediated SRC family kinase activation. J Exp Med. 2017;214(4):1049-64. https://doi. org/10.1084/jem.20160311.

16. Fantin A, Vieira JM, Gestri G, Denti L, Schwarz Q, Prykhozhij S, et al. Tissue macrophages act as cellular chaperones for vascular anastomosis downstream of VEGF-mediated endothelial tip cell induction. Blood. 2010; 116(5):829-40. https://doi.org/10.1182/blood-2009-12-257832.

17. Frank RN, Amin RH, Eliott D, Puklin JE, Abrams GW. Basic fibroblast growth factor and vascular endothelial growth factor are present in epiretinal and choroidal neovascular membranes. Am J Ophthalmol. 1996;122(3):393-403. https://doi.org/10.1016/S0002-9394(14)72066-5.

18. Fruttiger M. Development of the retinal vasculature. Angiogenesis. 2007; 10(2):77-88. https://doi.org/10.1007/s10456-007-9065-1.

19. Goldmann T, Wieghofer P, Jordão MJC, Prutek F, Hagemeyer N, Frenzel K, et al. Origin, fate and dynamics of macrophages at central nervous system interfaces. Nat Immunol. 2016;17(7):797-805. https://doi.org/10.1038/ni.3423.

20. Gupta N, Brown KE, Milam AH. Activated microglia in human retinitis pigmentosa, late-onset retinal degeneration, and age-related macular degeneration. Exp Eye Res. 2003;76(4):463-71. https://doi.org/10.1016/50014-4835(02)00332-9.

21. Hagemeyer N, Kierdorf K, Frenzel K, Xue J, Ringelhan M, Abdullah Z, et al. Transcriptome-based profiling of yolk sac-derived macrophages reveals a role for Irf8 in macrophage maturation. EMBO J. 2016;35(16):1730-44. https://doi.org/10.15252/embj.201693801.

22. Härdtner C, Kornemann J, Krebs K, Ehlert CA, Jander A, Zou J, et al. Inhibition of macrophage proliferation dominates plaque regression in response to cholesterol lowering. Basic Res Cardiol. 2020;115(6):78. https:// doi.org/10.1007/s00395-020-00838-4.
23. Haynes SE, Hollopeter G, Yang G, Kurpius D, Dailey ME, Gan W-B, et al. The P2Y12 receptor regulates microglial activation by extracellular nucleotides. Nat Neurosci. 2006;9(12):1512-9. https://doi.org/10.1038/nn1805.

24. Holtschke T, Löhler J, Kanno Y, Fehr T, Giese N, Rosenbauer F, et al. Immunodeficiency and chronic myelogenous leukemia-like syndrome in mice with a targeted mutation of the ICSBP gene. Cell. 1996;87(2):307-17. https://doi.org/10.1016/S0092-8674(00)81348-3.

25. Horiuchi M, Wakayama K, Itoh A, Kawai K, Pleasure D, Ozato K, et al. Interferon regulatory factor 8/interferon consensus sequence binding protein is a critical transcription factor for the physiological phenotype of microglia. J Neuroinflammation. 2012;9:227.

26. Jung S, Aliberti J, Graemmel P, Sunshine MJ, Kreutzberg GW, Sher A, et al. Analysis of fractalkine receptor CX(3)CR1 function by targeted deletion and green fluorescent protein reporter gene insertion. Mol Cell Biol. 2000;20(11): 4106-14. https://doi.org/10.1128/MCB.20.11.4106-4114.2000.

27. Kierdorf K, Erny D, Goldmann T, Sander V, Schulz C, Perdiguero EG, et al. Microglia emerge from erythromyeloid precursors via Pu.1- and Irf8dependent pathways. NatNeurosci. 2013;16:273-80.

28. Kobayakawa K, Ohkawa Y, Yoshizaki S, Tamaru T, Saito T, Kijima K, et al. Macrophage centripetal migration drives spontaneous healing process after spinal cord injury. Sci Adv. 2019;5:eaav5086.

29. Koso H, Nishinakamura R, Watanabe S. Sall1 regulates microglial morphology cell autonomously in the developing retina. Adv Exp Med Biol. 2018;1074:209-15. https://doi.org/10.1007/978-3-319-75402-4_26.

30. Kronlage M, Song J, Sorokin L, Isfort K, Schwerdtle T, Leipziger J, et al. Autocrine purinergic receptor signaling is essential for macrophage chemotaxis. Sci Signal. 2010;3:ra55.

31. Lange C, Mowat F, Sayed H, Mehad M, Duluc L, Piper S, et al. Dimethylarginine dimethylaminohydrolase-2 deficiency promotes vascular regeneration and attenuates pathological angiogenesis. Exp Eye Res. 2016; 147:148-55. https://doi.org/10.1016/j.exer.2016.05.007.

32. Lange CAK, Luhmann UFO, Mowat FM, Georgiadis A, West EL, Abrahams S, et al. Von Hippel-Lindau protein in the RPE is essential for normal ocular growth and vascular development. Dev Camb Engl. 2012;139:2340-50.

33. Lavin Y, Winter D, Blecher-Gonen R, David E, Keren-Shaul H, Merad M, et al. Tissue-resident macrophage enhancer landscapes are shaped by the local microenvironment. Cell. 2014;159(6):1312-26. https://doi.org/10.1016/j.cell.2 014.11.018.

34. Liu J, Copland DA, Horie S, Wu W-K, Chen M, Xu Y, et al. Myeloid cells expressing VEGF and arginase-1 following uptake of damaged retinal pigment epithelium suggests potential mechanism that drives the onset of choroidal angiogenesis in mice. PloS One. 2013;8(8):e72935. https://doi. org/10.1371/journal.pone.0072935.

35. Liyanage SE, Fantin A, Villacampa P, Lange CA, Denti L, Cristante E, et al. Myeloid-derived vascular endothelial growth factor and hypoxia-inducible factor are dispensable for ocular neovascularization-brief report. Arterioscler Thromb Vasc Biol. 2016;36(1):19-24. https://doi.org/10.1161/ATVBAHA.115.3 06681.

36. Lückoff A, Caramoy A, Scholz R, Prinz M, Kalinke U, Langmann T. Interferonbeta signaling in retinal mononuclear phagocytes attenuates pathological neovascularization. EMBO Mol Med. 2016;8(6):670-8. https://doi.org/10.152 52/emmm.201505994.

37. Martín-Estebané M, Navascués J, Sierra-Martín A, Martín-Guerrero SM, Cuadros MA, Carrasco M-C, et al. Onset of microglial entry into developing quail retina coincides with increased expression of active caspase- 3 and is mediated by extracellular ATP and UDP. PloS One. 2017;12(8):e0182450. https://doi.org/10.1371/journal.pone.0182450.

38. Masuda T, Amann L, Sankowski R, Staszewski O, Lenz M, Errico PD, et al. Novel Hexb-based tools for studying microglia in the CNS. Nat Immunol. 2020;21(7):802-15. https://doi.org/10.1038/s41590-020-0707-4.

39. Masuda T, Iwamoto S, Mikuriya S, Tozaki-Saitoh H, Tamura T, Tsuda M, et al. Transcription factor IRF1 is responsible for IRF8-mediated IL-1 3 expression in reactive microglia. J Pharmacol Sci. 2015;128(4):216-20. https://doi.org/10.1016/.jphs.2015.08.002.

40. Masuda T, Nishimoto N, Tomiyama D, Matsuda T, Tozaki-Saitoh H, Tamura T, et al. IRF8 is a transcriptional determinant for microglial motility. Purinergic Signal. 2014;10(3):515-21. https://doi.org/10.1007/s11302-014-9413-8.

41. Matsuda Y, Nonaka Y, Futakawa S, Imai H, Akita K, Nishihata T, et al. Antiangiogenic and anti-scarring dual action of an anti-fibroblast growth factor 2 aptamer in animal models of retinal disease. Mol Ther Nucleic Acids. 2019; 17:819-28. https://doi.org/10.1016/j.omtn.2019.07.018. 
42. Miller CG, Budoff G, Prenner JL, Schwarzbauer JE. Minireview: fibronectin in retinal disease. Exp Biol Med Maywood NJ. 2017;242(1):1-7. https://doi.org/1 $0.1177 / 1535370216675245$.

43. Miller CG, Pozzi A, Zent R, Schwarzbauer JE. Effects of high glucose on integrin activity and fibronectin matrix assembly by mesangial cells. Mol Biol Cell. 2014:25(16):2342-50. https://doi.org/10.1091/mbc.e14-03-0800.

44. Minten C, Terry R, Deffrasnes C, King NJC, Campbell IL. IFN regulatory factor 8 is a key constitutive determinant of the morphological and molecular properties of microglia in the CNS. PloS One. 2012;7(11):e49851. https://doi. org/10.1371/journal.pone.0049851.

45. Nimmerjahn A, Kirchhoff F, Helmchen F. Resting microglial cells are highly dynamic surveillants of brain parenchyma in vivo. Science. 2005;308(5726): 1314-8. https://doi.org/10.1126/science.1110647.

46. O'Koren EG, Mathew R, Saban DR. Fate mapping reveals that microglia and recruited monocyte-derived macrophages are definitively distinguishable by phenotype in the retina. Sci Rep. 2016;6(1):20636. https://doi.org/10.1038/ srep20636.

47. Prinz M, Jung S, Priller J. Microglia biology: one century of evolving concepts. Cell. 2019;179(2):292-311. https://doi.org/10.1016/j.cell.2019.08.053.

48. Prinz M, Masuda T, Wheeler MA, Quintana FJ. Microglia and central nervous system-associated macrophages-from origin to disease modulation. Annu Rev Immunol. 2021;39(1):251-77. https://doi.org/10.1146/annurev-immunol093019-110159.

49. Rashid K, Akhtar-Schaefer I, Langmann T. Microglia in retinal degeneration. Front Immunol. 2019;10:1975. https://doi.org/10.3389/fimmu.2019.01975.

50. Robbie SJ, Lundh von Leithner P, Ju M, Lange CA, King AG, Adamson P, et al. Assessing a novel depot delivery strategy for noninvasive administration of VEGF/PDGF RTK inhibitors for ocular neovascular disease. Invest Ophthalmol Vis Sci. 2013;54(2):1490-500. https://doi.org/10.1167/ iovs.12-10169.

51. Sakurai E, Anand A, Ambati BK, Van RN, Ambati J. Macrophage depletion inhibits experimental choroidal neovascularization. Invest Ophthalmol VisSci. 2003;44(8):3578-85. https://doi.org/10.1167/iovs.03-0097.

52. Schlecht A, Zhang P, Wolf J, Thien A, Rosmus D-D, Boneva S, et al. Secreted phosphoprotein 1 expression in retinal mononuclear phagocytes links murine to human choroidal neovascularization. Front Cell Dev Biol. 2021;8: 618598.

53. Schönheit J, Kuhl C, Gebhardt ML, Klett FF, Riemke P, Scheller M, et al. PU.1 level-directed chromatin structure remodeling at the Irf8 gene drives dendritic cell commitment. Cell Rep. 2013;3(5):1617-28. https://doi.org/10.1 016/j.celrep.2013.04.007.

54. Schwarzer P, Kokona D, Ebneter A, Zinkernagel MS. Effect of inhibition of colony-stimulating factor 1 receptor on choroidal neovascularization in mice. Am J Pathol. 2020;190(2):412-25. https://doi.org/10.1016/j.ajpath.201 9.10.011.

55. Shemer A, Grozovski J, Tay TL, Tao J, Volaski A, Süß P, et al. Engrafted parenchymal brain macrophages differ from microglia in transcriptome, chromatin landscape and response to challenge. Nat Commun. 2018;9:5206.

56. Silverman SM, Wong WT. Microglia in the retina: roles in development, maturity, and disease. Annu Rev Vis Sci. 2018;4(1):45-77. https://doi.org/1 0.1146/annurev-vision-091517-034425

57. Tamura T, Kurotaki D, Koizumi S-I. Regulation of myelopoiesis by the transcription factor IRF8. Int J Hematol. 2015;101(4):342-51. https://doi.org/1 0.1007/s12185-015-1761-9.

58. Van Hove H, Martens L, Scheyltjens I, De Vlaminck K, Pombo Antunes AR, De Prijck $S$, et al. A single-cell atlas of mouse brain macrophages reveals unique transcriptional identities shaped by ontogeny and tissue environment. Nat Neurosci. 2019;22(6):1021-35. https://doi.org/10.1038/s41 593-019-0393-4.

59. Wieghofer P, Hagemeyer N, Sankowski R, Schlecht A, Staszewski O, Amann $L$, et al. Mapping the origin and fate of myeloid cells in distinct compartments of the eye by single-cell profiling. EMBO J. 2021;40:e105123.

60. Wong WL, Su X, Li X, Cheung CMG, Klein R, Cheng C-Y, et al. Global prevalence of age-related macular degeneration and disease burden projection for 2020 and 2040: a systematic review and meta-analysis. Lancet Glob Health. 2014; 2(2):e106-16. https:/doi.org/10.1016/S2214-109X(13)70145-1.
61. Yoshida Y, Yoshimi R, Yoshii H, Kim D, Dey A, Xiong $H$, et al. The transcription factor IRF8 activates integrin-mediated TGF- $\beta$ signaling and promotes neuroinflammation. Immunity. 2014;40(2):187-98. https://doi.org/1 0.1016/j.immuni.2013.11.022

62. Yu G, Wang L-G, Han Y, He Q-Y. clusterProfiler: an R package for comparing biological themes among gene clusters. OMICS J Integr Biol. 2012;16(5):2847. https://doi.org/10.1089/omi.2011.0118.

\section{Publisher's Note}

Springer Nature remains neutral with regard to jurisdictional claims in published maps and institutional affiliations.
Ready to submit your research? Choose BMC and benefit from:

- fast, convenient online submission

- thorough peer review by experienced researchers in your field

- rapid publication on acceptance

- support for research data, including large and complex data types

- gold Open Access which fosters wider collaboration and increased citations

- maximum visibility for your research: over $100 \mathrm{M}$ website views per year

At BMC, research is always in progress.

Learn more biomedcentral.com/submissions 